$\mathbb{T}$ periodica polytechnica

Chemical Engineering

$56 / 2$ (2012) 65,69

doi: $10.3311 /$ pp.ch.2012-2.03

web: http://www.pp.bme.hu/ch

(c) Periodica Polytechnica 2012

RESEARCH ARTICLE

\section{Influence of breakage on crystal size distribution in a continuous cooling crystallizer}

\author{
Ákos Borsos / Béla G. Lakatos
}

Received 2012-05-15, accepted 2012-10-09

\begin{abstract}
A detailed two-dimensional population balance model of continuous cooling crystallization, involving nucleation, growth of the two characteristic crystal facets and binary breakage along the length of needle-shape crystals is presented and analysed. The population balance equation is reduced into a moment equation model of the joint moments of crystal size variables. The dynamic behaviour of the crystallizer and the effects of $\mathrm{ki}$ netic and process parameters on the characteristics of crystal size distribution are studied by simulation. The observations and analysis have revealed that there exist strong interactions between the breakage and the product properties.
\end{abstract}

\section{Keywords}

Crystallization · population balance model $\cdot$ crystal breakage $\cdot$ moment method $\cdot$ simulation

\section{Acknowledgement}

This work was presented at the Conference of Chemical Engineering, Veszprém, 2012.

This work was supported by the Hungarian Scientific Research Fund under Grant K77955 which is gratefully acknowledged. The financial support of the TAMOP-4.2.1/B-09/1/KONV2010-0003 project is also acknowledged.

\footnotetext{
Ákos Borsos

Department of Process Engineering, University of Pannonia, H-8200 Veszprém, Egyetem Street 10, Hungary

e-mail: borsosa@fmt.uni-pannon.hu
}

Béla G. Lakatos

Department of Process Engineering, University of Pannonia, H-8200 Veszprém, Egyetem Street 10, Hungary

\section{Introduction}

Crystallization is an important unit operation of chemical and process industries and it is a suitable method of formulation of solid particles, separation and purification of chemical components. There exist special demands in industrial practice for well-designed crystalline products which require developing more precise operation methods. Batch cooling crystallization is an often used method in industry, especially in the pharmaceutical industry hence well designed and operated batch processes seem to have great advantage in producing appropriately tailored crystalline products. Naturally, the detailed knowledge of these processes provides an elementary requirement for success.

Crystallization is a complex process which contains many basic processes such as primary and secondary nucleation and growth of crystals, but crystal breakage and agglomeration can often be observed in crystallizers. The breakage of crystals may play especially important role in solution crystallization when crystals are characterized by non-isometric crystal habits. The high aspect ratio crystals, i.e. needle-shape or rod-like crystals which often are met in the pharmaceutical industry, possess, among others, such habits therefore investigation of the breakage process of those crystals seems to be of fundamental importance.

Biscans [1] studied the breakage of mono sodium glutamate crystals; Bao et al. [2] presented a model of L-threonine crystals describing their growth and binary breakage. Population balance models were applied by Sato et al. [3] and Grof et al. [4] to characterise the breakage phenomenon of high aspect ratio crystals. Ma and Wang [5] determined the facet growth kinetics of L-glutamic acid crystals using in-process image analysis.

2D population balance models were applied by Ma et al. [6] for simulation of crystallization of KDP crystals, and by Puel et al. [7] for batch crystallization of a rod-like organic product. Briesen [8] developed a modified moment method for reducing a 2D population balance model of crystallization while Borsos [9], Lakatos [10], and Borsos and Lakatos [11] applied a 2D moment method to investigate the problem.

The aim of this work is to present and analyse a detailed 2D population balance for a continuous cooling crystallization of 
high aspect ratio crystals with their possible fragmentation by using the standard moment method. The two dimensional population balance equation which is suitable to model non-isometric crystal habits such as high aspect ratio crystals is extended with breakage terms and completed with the mass and energy balance equations. Then it is converted into a set of moment equations for the joint moments of the crystal sizes. The results of this process are compared with the system without breakage by using numerical simulators which were developed in Matlab environment.

\section{Population balance model}

Crystals with needle-like habits can be characterised by two size dimensions $L_{1}$ and $L_{2}$, which are sufficient to compute the volumes of crystals, required to develop the mass and heat balance equations for crystallizers [10].

In this case the crystal population is described by the $2 \mathrm{D}$ population density function $\left(L_{1}, L_{2}, t\right) \rightarrow n\left(L_{1}, L_{2}, t\right)$ by means of which $n\left(L_{1}, L_{2}, t\right) \mathrm{d} L_{1} \mathrm{~d} L_{2}$ expresses the number of crystals from the size domain $\left(L_{1}, L_{1}+\mathrm{d} L_{1}\right) \times\left(L_{2}, L_{2}+\mathrm{d} L_{2}\right)$ in a unit volume of suspension at time $t$.

Let us now assume that:

- The working volume of the crystallizer is constant;

- All new crystals are formed at a nominal size $L_{1, n} \approx L_{2, n} \approx$ $L_{3, n} \approx L_{n} \geq 0$, so that we can assume: $L_{n} \approx 0$;

- Crystal agglomeration is negligible.

The kinetic processes can be described by the following equations. The primary nucleation rate is given as

$$
B_{p}=\varepsilon k_{p 0} \exp \left(-\frac{E_{p}}{R T}\right) \exp \left(-\frac{k_{e}}{\ln ^{2} S}\right)
$$

where $S=c / c_{s}$ denotes the supersaturation ratio, $c$ and $c_{s}$ denote, respectively, the solute and equilibrium saturation concentrations, and $\varepsilon$ stands for the volumetric ratio of solution. Moreover, $k_{i 0}$ is kinetic constant of primary nucleation; $E_{p}$ is the activation energy, $R$ is the gas constant, $T$ means the temperature and $k_{e}$ is a parameter of primary nucleation. The rate of secondary nucleation is

$$
B_{b}=k_{b 0} \exp \left(-\frac{E_{b}}{R T}\right) \sigma^{b} \mu_{1,2}^{j}
$$

where $\sigma=\left(c-c_{s}\right) / c_{s}$ is the relative supersaturation and $\mu_{1,2}$ stands for the third order joint moment.

The size independent growth rate is given by the following equation:

$$
G_{i}=k_{g i 0} \exp \left(-\frac{E_{g i}}{R T}\right) \sigma^{g_{1}}
$$

where $i=1,2$ and the kinetic coefficients are constant.

Then the population balance equation contains breakage parts and there are two important equations of those, as the selection function

$$
S_{b r}^{1}\left(L_{1}, L_{2}\right)=k_{\text {break }} L_{1}^{\beta} L_{2}^{\gamma}
$$

where $\beta$ and $\gamma$ are the constant exponents of sizes.

The second function characterizing a breakage event which is termed breakage function provides the fragment sizes of the broken particle. In this case the following form is applied

$$
b_{b r}^{1}\left(L_{1}, \lambda_{1}\right) b_{b r}^{2}\left(L_{2}, \lambda_{2}\right)=2 \delta\left(L_{1}-\frac{\lambda_{1}}{2}\right) \delta\left(L_{2}-\lambda_{2}\right)
$$

where $\delta$ is delta function and $\lambda$ means the sizes of mother crystals.

These two equations present that the crystals can break up along the two different sizes, but in this study we assumed that $\gamma=0$ which means that breakage occurs only along the length of crystals.

Then the population balance equation with breakage could be given as the follows.

$$
\begin{aligned}
& \frac{\partial n\left(L_{1}, L_{2}, t\right)}{\partial t}+\frac{\partial\left[G_{1} n\left(L_{1}, L_{2}, t\right)\right]}{\partial L_{1}}+\frac{\partial\left[G_{2} n\left(L_{1}, L_{2}, t\right)\right]}{\partial L_{2}}= \\
& \frac{1}{\tau}\left[n_{\text {in }}\left(L_{1}, L_{2}, t\right)-n\left(L_{1}, L_{2}, t\right)\right] \\
& -k_{\text {break }} \int_{0}^{L_{m}} \int_{0}^{L_{m}} \delta\left(\lambda_{1}-\frac{L_{1}}{2}\right) \delta\left(\lambda_{2}-L_{2}\right) L_{1}^{\beta} n\left(L_{1}, L_{2}, t\right) d \lambda_{1} d \lambda_{2} \\
& +k_{\text {break }} \int_{0}^{L_{m}} \int_{0}^{L_{m}} 2 \delta\left(L_{1}-\frac{\lambda_{1}}{2}\right) \delta\left(L_{2}-\lambda_{2}\right) \lambda_{1}^{\beta} n\left(\lambda_{1}, \lambda_{2}, t\right) d \lambda_{1} d \lambda_{2}
\end{aligned}
$$

where $n$ is population density function and $\tau$ is the mean residence time.

Here are the initial and boundary conditions

$$
n\left(L_{1}, L_{2}, t=0\right)=n_{0}\left(L_{1}, L_{2}\right)
$$

$$
\begin{array}{ll}
\lim _{\substack{L_{1} \rightarrow 0 \\
L_{2} \rightarrow 0}} & {\left[G_{1} n\left(L_{1}, L_{2}, t\right)+G_{2} n\left(L_{1}, L_{2}, t\right)\right]=} \\
& e_{p} B_{p}\left(L_{1}, L_{2}, t\right)+e_{b} B_{b}\left(L_{1}, L_{2}, t\right) \\
& \\
& \lim _{\substack{L_{1} \rightarrow \infty \\
L_{2} \rightarrow \infty}} n\left(L_{1}, L_{2}, t\right)=0
\end{array}
$$

where $e_{p}$ and $e_{b}$ are binary existence variables by means of which an appropriate combination of the primary and secondary nucleation rates can be given.

\section{Moment method}

The properties of crystalline particles in the crystallization process and behaviour of the crystallizer are determined by the population balance model but the numerical solution of Eq. (6) is a complex procedure. As it was mentioned earlier, the moment method is able to calculate the properties of the crystallization process. This method is widely used in modelling of disperse systems. Developing the mass and heat balance equations for the crystallizer requires an expression for the total mass of crystal population. It has to be expressed by means of the volume of 
a single crystal computed by means of their two identified sizes $L_{1}$ and $L_{2}$.

The partial volume of crystals in the suspension is given as

$$
1-\varepsilon(t)=\iint_{L} L_{1} L_{2}^{2} n\left(L_{1}, L_{2}, t\right) d L_{1} d L_{2}=\mu_{1,2}
$$

where $v_{c}(t)=L_{1} L_{2}^{2}$ denotes the volume of single crystals.

In this $2 \mathrm{D}$ case, the infinite system of moment equations takes the form

$$
\begin{aligned}
& \frac{d \mu_{0,0}}{d t}=\frac{1}{\tau}\left(\mu_{0,0, i n}-\mu_{0,0}\right)+e_{p} B_{p}+e_{b} B_{b}+k_{\text {break }}(\varepsilon) \mu_{\beta, m} \\
& \frac{d \mu_{k, m}}{d t}=\frac{1}{\tau}\left(\mu_{k, m, i n}-\mu_{k, m}\right)+k G_{1} \mu_{k-1, m}+m G_{2} \mu_{k, m-1}+ \\
& \left(\frac{1}{2^{k-1}}-1\right) k_{\text {break }}(\varepsilon) \mu_{k+\beta, m} \\
& k, m=0,1,2,3 \ldots, \quad k+m>0
\end{aligned}
$$

which can be simply closed when $\beta=0$ or $\beta=1$ and extended this model with the mass and energy balances. The mass balance equation of solute has the form

$$
\frac{d c}{d t}=\frac{\varepsilon_{i n}}{\tau \varepsilon}\left(c_{i n}-c\right)-\frac{\left(\rho_{c}-c\right)}{\varepsilon} R_{V}
$$

where $R_{V}$ denotes the rate of change of the total volume of crystals in a unit volume of suspension. Then the mass balance of solvent is the following

$$
\frac{d c_{s v}}{d t}=\frac{\varepsilon_{i n}}{\tau \varepsilon}\left(c_{s v i n}-c_{s v}\right)+\frac{c_{s v}}{\varepsilon} R_{V}
$$

The energy balance equation for the crystal suspension is

$$
\frac{d T}{d t}=\frac{\Theta_{i n}}{\tau \Theta}\left(T_{i n}-T\right)-\frac{U a_{V}}{\Theta}\left(T-T_{h}\right)+\frac{\left(-\Delta H_{c}\right)}{\Theta} \rho_{c} R_{V}
$$

where $\Theta=\varepsilon\left(C_{s v} c_{s v}+C_{c} c\right)+(1-\varepsilon) C_{c} \rho, U$ is the heat transfer coefficient, $a_{V}$ denotes the surface of heat transfer, $\left(-\Delta H_{c}\right)$ stands for the heat of crystallization, while $C_{c}$ and $C_{s v}$ are the specific heat of solute and solvent.

The energy balance of cooling medium takes the form

$$
\frac{d T}{d t}=\frac{1}{\tau_{h}}\left(T_{h i n}-T_{h}\right)+\beta_{h}\left(T-T_{h}\right)
$$

The equation system of the Eq. (10)-Eq. (14) is a closed differential equation system. Thus it is suitable model to make dynamic calculations on the crystallizer.

\section{Simulation and results}

Numerical solution of the set of ordinary differential equations was carried out in MATLAB environment. The basic values of the process parameters are presented in Table 1 while the basic values of kinetic parameters of nucleation, crystal growth and breakage used in simulation are listed in the Table 2 [3].

The breakage events depend on the parameter $k_{\text {break }}$ [12]. In this study, the system was investigated by using a simulator with
Tab. 1. Basic values of process parameters used in simulation

\begin{tabular}{ll}
\hline$V=1.010^{-3} \mathrm{~m}^{3}$ & $\tau=10^{3} \mathrm{~s}$ \\
\hline$T_{i n}=90^{\circ} \mathrm{C}$ & $T_{h}=20{ }^{\circ} \mathrm{C}$ \\
\hline$\tau_{h}=6 \cdot 10^{2} \mathrm{~s}$ & $\beta_{h}=2.0 \cdot 10^{-2}$ \\
\hline$U a_{V}=5.0 \cdot 10^{5}$ & $\varphi_{\text {in }}=3.0 \cdot 10^{6}$ \\
\hline
\end{tabular}

Tab. 2. Basic values of kinetic parameters used in simulation

\begin{tabular}{lll}
\hline$k_{b 0}=2.0 \cdot 10^{7} \# \mathrm{~m}^{-3} \mathrm{~s}^{-1}$ & $k_{p 0}=1.6 \cdot 10^{18} \#^{-3} \mathrm{~s}^{-1}$ & $g_{1}=1.5$ \\
\hline$k_{g 2}=1.0 \cdot 10^{-3} \mathrm{~m} \mathrm{~s}^{-1}$ & $k_{g 1}=1.0 \cdot 10^{-4} \mathrm{~m} \mathrm{~s}^{-1}$ & $g_{2}=1.75$ \\
\hline$b=2.0$ & $k_{e}=1.0$ & $\Delta H_{c}=-44.5 \mathrm{~J} \mathrm{~kg}^{-1}$ \\
\hline$E_{b}=1.5 \cdot 10^{4}$ & $E_{g}=3 \cdot 10^{4}$ & $E_{p}=1 \cdot 10^{4}$ \\
\hline$a_{1}=-9.7629 \mathrm{e}-5$ & $a_{0}=0.2087$ & $a_{2}=9.3027 \mathrm{e}-5$ \\
\hline$\beta=1$ & $j=1.5$ & \\
\hline
\end{tabular}

two different rates of this parameter. In the first case $k_{\text {break }}=0$ was assumed which means that there is no breakage during the process and in the other case we assumed $k_{\text {break }}=40$. That means an intensive breakage rate.

Fig. 1 presents the temporal evolutions of temperatures of the crystalline suspension and cooling medium. There are peaks on the diagrams which mean that nucleation heats up the system. On the applied initial level of saturation ratio the nucleation starts instantly which is the reason of large increase of heat.

Fig. 2 presents the temporal evolutions of the solute and the solubility concentrations. At the beginning, the differences between the two values of concentrations are significant and then it decreases, but the state of the crystallizer becomes always supersaturated.

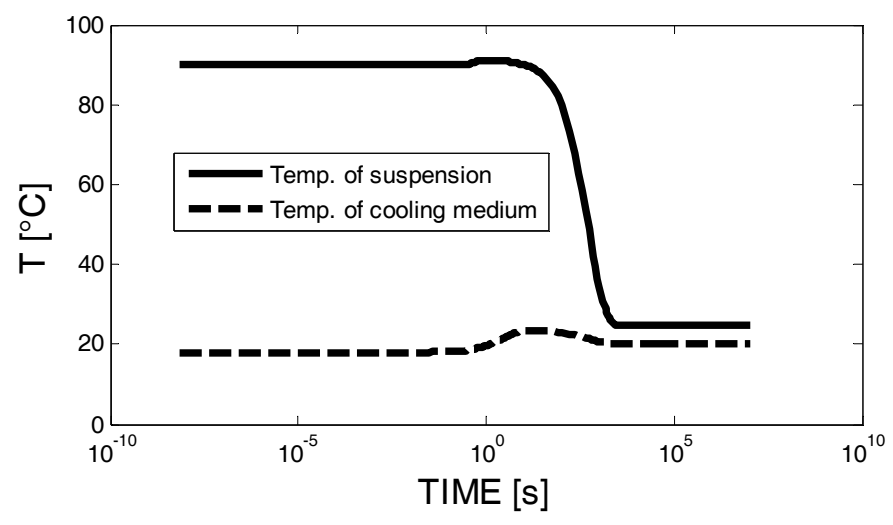

Fig. 1. Temperature profile in the suspension and in the cooling medium

Fig. 3 and Fig. 4 present the temporal evolutions of the two mean sizes $\left\langle L_{1}\right\rangle$ and $\left\langle L_{2}\right\rangle$.

The breakage process in this case causes decrease of the steady state mean crystal length slightly while the steady state mean width of crystals increases when breakage occurs. The reason of this phenomenon is that the breakage process is size dependent with parameter $\beta=1$.

Fig. 5 presents evolutions in time of the zero order moment 


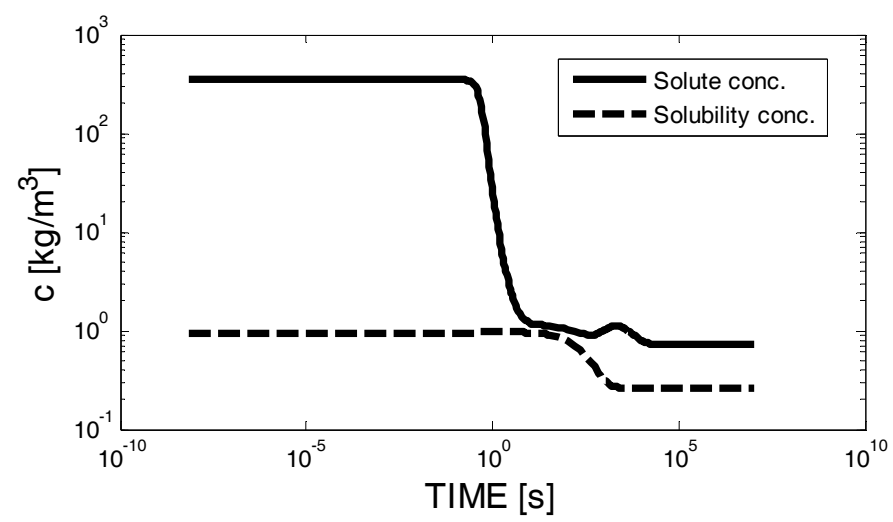

Fig. 2. Evolution in time of the solute- and solubility concentration

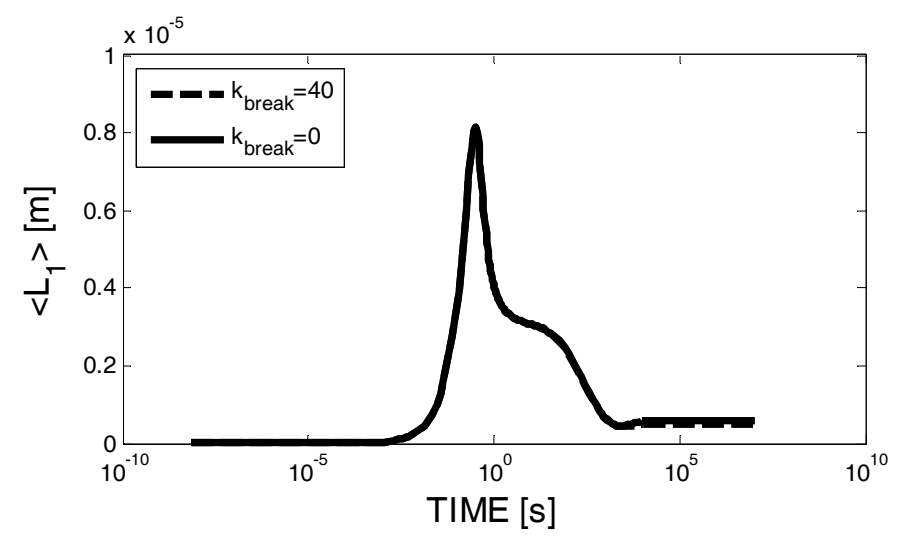

Fig. 3. Evolution of the mean length $\left(L_{1}\right)$ in time

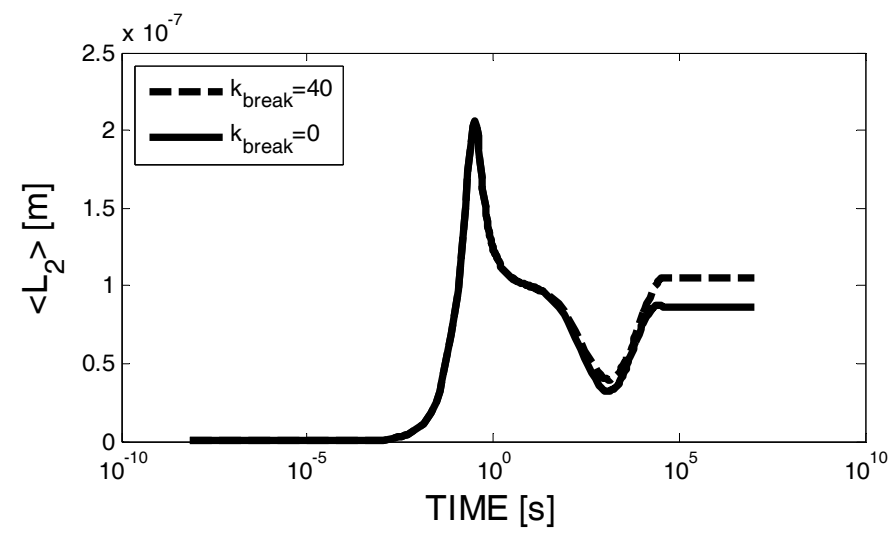

Fig. 4. Evolution of the mean width $\left(L_{2}\right)$ in time

$\mu_{00}$ and illustrates how the total number of particles depends on the breakage rate. Naturally, the breakage process produces more particles in steady state compared with that without breakage but this increase becomes significant only when the nucleation process is terminated. The maxima in these time diagrams arise because of the differences of the characteristic times of crystal production and crystallizer, i.e. its mean residence time.

The third order joint moment $\mu_{12}$ relates to the total volume of solid particles. Thus Fig. 6 shows that the total crystal volume does not differ in the observed cases since breakage of crystals does not influence the total volume and total mass of the crystalline product.

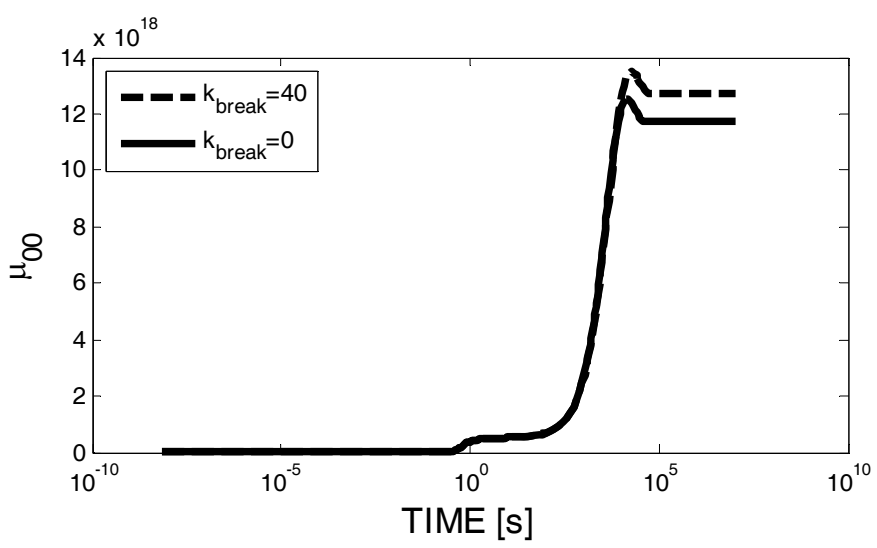

Fig. 5. Evolution of the zero order moment $\mu_{0,0}$ in time

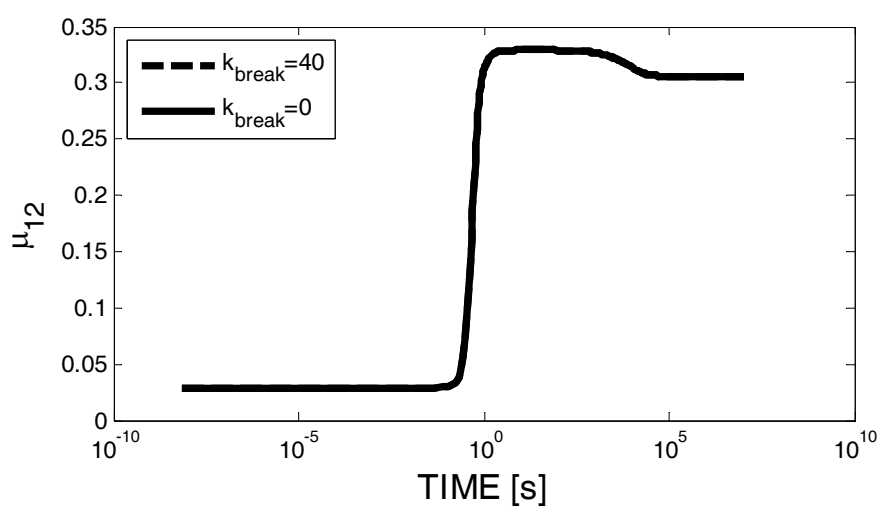

Fig. 6. Temporal evolution of the third order moment $\mu_{1,2}$ of the crystalline product

\section{Conclusions}

A detailed two-dimensional population balance model was presented for describing continuous cooling crystallization of needle-shape crystals with fragmentation. The model contains nucleation, growth and breakage of crystals. The closed set of moment equations of the joint moments of the crystal size variables with the mass and energy balance equations made possible of computing the dynamic properties of the crystallizer.

The numerical analysis revealed that there exist strong interactions between the nucleation, growth and breakage processes of needle-shape crystals and made an opportunity to study the behaviour of a crystallization system with non-regular properties such as high aspect ratio crystals. 


\section{Symbols}

$b$ exponent of secondary nucleation rate

$B \quad$ nucleation rate $\# \mathrm{~m}^{-3} \mathrm{~s}^{-1}$

c concentration of solute, $\mathrm{kgm}^{-3}$

$c_{s} \quad$ equilibrium saturation concentration, $\mathrm{kg} \mathrm{m}^{-3}$

$E_{i} \quad$ activation energy $(i=b, p, g), \mathrm{kJ} \mathrm{kmol}^{-1}$

$g \quad$ exponent of crystal growth rate

$G \quad$ crystal growth rate, $\mathrm{ms}^{-1}$

$j \quad$ exponent of secondary nucleation rate

$k_{\text {break }}$ rate coefficient of breakage, $1 \mathrm{~m}^{-(2+\beta)} \mathrm{s}^{-1}$

$k_{e} \quad$ parameter of primary nucleation rate

$k_{g} \quad$ rate coefficient of crystal growth, $\mathrm{m} \mathrm{s}^{-1}$

$k_{p} \quad$ rate coefficient of primary nucleation, $\# \mathrm{~m}^{-3} \mathrm{~s}^{-1}$

$k_{b} \quad$ rate coefficient of secondary nucleation, $\# \mathrm{~m}^{-3} \mathrm{~s}^{-1}$

$k_{V} \quad$ volume shape factor

$L \quad$ linear size of crystals, $\mathrm{m}$

$n \quad$ population density function, $\# \mathrm{~m}^{-5}$

$R \quad$ gas constant

$S \quad$ supersaturation ratio, $\mathrm{c} / \mathrm{c}_{s}$

T temperature, ${ }^{\circ} \mathrm{C}, \mathrm{K}$

\section{Greek letters:}

$\beta \quad$ breakage parameter

$\beta_{h} \quad$ eq. 14 .

$\varepsilon \quad$ volumetric ratio of solution

$\mu_{k, m} \quad(\mathrm{k}, \mathrm{m})^{t h}$ order joint moment

$\rho$ density, $\mathrm{kgm}^{-3}$

$\tau \quad$ mean residence time, $\mathrm{s}$

$\tau_{h} \quad$ mean residence time of the cooling medium, $\mathrm{s}$

$\sigma \quad$ relative supersaturation

\section{Subscripts:}

0 initial value

1 length coordinate of crystals, $m$

2 width coordinate of crystals, $m$

in inlet value

$p$ primary nucleation

$b$ secondary nucleation

$h$ cooling medium

\section{References}

1 Biscans B, Impact attrition in crystallization processes. Analysis of repeated impacts events of individual crystals, Powder Technology 143-144 (2004), 264-272, DOI 10.1016/j.powtec.2004.04.025.

2 Bao Y, Zhang J, Yin Q, Wang J, Determination of growth and breakage kinetics of L-threonine crystals, Journal of Crystal Growth 289 (2006), 317 323, DOI 10.1016/j.jcrysgro.2005.11.001.

3 Sato K, Nagai H, Hasegawa K, Tomori K, Kramer H J M, Jansens $\mathbf{P} \mathbf{J}$, Two-di-mensional population balance model with breakage of high aspect ratio crystals for batch crystallization, Chemical Engineering Science 63 (2008), 3271-3278, DOI 10.1016/j.ces.2008.03.013.

4 Grof Z, Schoellhammer C M, Rajniak P, Stepanek F, Computational and experimental investigation of needle-shaped crystal breakage., International Journal of Pharmaceutics 407 (2011), 12-20, DOI 10.1016/j.ijpharm.2010.12.031.

5 Ma C Y, Wang X Z, Model identification of crystal facet growth kinetics in morphological population balance modelling of L-glutamic acid crystallization and experimental validation, Chemical Engineering Science, posted on 2011, DOI 10.1016/j.ces.2011.05.042., (to appear in print).

6 Ma D L, Tafti D K, Braatz R D, High-resolution simulation of multidimensional crystal growth, Industrial \& Engineering Chemistry Research 41 (2002), 6217-6223, DOI 10.1021/ie010680u.

7 Puel F, Févotte G, Klein J P, Simulation and analysis of industrial crystallization processes through multidimensional population balance equations, Chemical Engineering Science 58 (2003), 3715-3727, 3729-3740, DOI 10.1016/S0009-2509(03)00253-7.

8 Briesen $\mathbf{H}$, Simulation of crystal size and shape by means of a reduced twodimensional population balance model, Chemical Engineering Science 61 (2006), 104-112, DOI 10.1016/j.ces.2004.11.062.

9 Borsos Á, Modelling and optimization of crystallization processes, University of Pannonia, Hungary, 2009. MSc Thesis.

10 Lakatos B G, Modeling 2D crystal shape in cooling crystallization by moment method, Proceedings 4th International Conference on Population Balance Modelling, 2010, pp. 885-903.

11 Borsos Á, Lakatos B G, Modelling 2D Crystal Shape in Cooling Crystallization by Moment Method, Proceedings of InterTech 2011, 2011, pp. 224228.

12 Borsos Á, Lakatos B G, 2 D population balance model for crystallization of needle-shape crystals with fragmentation, Proc. of ISIC-18, Zurich, Switzerland, pp. 104-105. 